\title{
Modelo de educación en energías renovables desde el compromiso público y la actitud energética
}

\author{
Vladimir Alfonso Ballesteros-Ballesteros ${ }^{1}$ \\ Adriana Patricia Gallego-Torres ${ }^{2}$
}

Fecha de recepción: 10 de febrero de 2019

Fecha de aceptación: 17 de abril de 2019

\section{Resumen}

La crisis energética actual del planeta, es una realidad a lo que no podemos hacer la vista gorda. Ahora más que en otras épocas se agudizan los problemas energéticos, derivados producción, transformación, distribución y consumo de la energía que conllevó al evidente el agotamiento de los combustibles de origen fósil, a los problemas ambientales generados por la explotación, transporte y uso de los recursos naturales, el cambio climático, la contaminación, entre otros. El trabajo que aquí presentamos, tiene la intención principal de proponer un modelo conceptual basado en una construcción teórica a partir de una metodología mixta de análisis documental, desde la formulación de tres categorías: la educación en energías renovables (EER), el compromiso público (CP) y la conceptualización elaborada por nuestro equipo sobre actitud energética $(A E)$; todo esto sumado a las necesarias interacciones que se generan desde la democracia participativa y la construcción social comunitaria que pretende involucrar a la sociedad como estructura principal y no como un fin.

En segundo lugar, la importancia de contribuir al desarrollo del campo de investigación de la Educación en Energías Renovables, a razón de las discusiones que actualmente se plantean frente a la falta de responsabilidad social energética y el desinterés por el uso de energías alternativas como estrategia de reducción de

1 Ph. D. (c) Fundación Universitaria Los Libertadores (Bogotá-Distrito Capital, Colombia). vladimir.ballesteros@libertadores.edu.co. ORCID: 0000-0002-6920-789X.

2 Ph. D. Universidad Distrital Francisco José de Caldas (Bogotá-Distrito Capital, Colombia). adpgallegot@udistrital.edu.co. ORCID: 0000-0002-6654-3177. 
los problemas ambientales del contexto; por último, la necesidad de formular nuevos caminos para la implementación del uso de las energías renovables por parte de la ciudadanía con la intencionalidad de mitigar los problemas derivados de un desarrollo desmedido de la ciencia y la tecnología problemática que se ha ido en las últimas décadas.

Palabras clave: actitud energética; compromiso público; educación en energías renovables; modelo.

\section{Model of Education in Renewable Energies from the Public Engagement and the Energetic Attitude}

\section{Abstract}

The current energy crisis of the planet is a reality to which we cannot turn a blind eye. Now more than at other times, the energy problems are sharpened, resulting in the production, transformation, distribution and consumption of energy that led to the evident depletion of fossil fuels, environmental problems generated by the exploitation, transportation and use of natural resources. natural resources, climate change, pollution, among others. The work presented here, has the main intention of proposing a conceptual model based on a theoretical construction from a mixed methodology of documentary analysis, from the formulation of three categories: education in renewable energies (EER), public commitment (CP) and the conceptualization developed by our team on energy attitude (AE); all this added to the necessary interactions that are generated from participatory democracy and community social construction that aims to involve society as the main structure and not as an end.

Secondly, the importance of contributing to the development of the research field of Education in Renewable Energy, due to the discussions that currently arise in the face of the lack of social responsibility in energy and the disinterest in the use of alternative energies as a strategy of reduction of the environmental problems of the context; finally, the need to formulate new ways for the implementation of the use of renewable energies by citizens with the intention of mitigating the problems derived 
from an excessive development of science and technology that has been problematic in recent decades.

Keywords: education in renewable energy; energy attitude; model; public engagement.

\section{Modelo de educação em energias renováveis desde o compromisso público e a atitude energética}

\section{Resumo}

A crise energética atual do planeta, é uma realidade à qual não podemos simplesmente fechar os olhos. Agora mais que em outras épocas se agudizam os problemas energéticos, derivados da produção, transformação, distribuição e consumo da energia, que levou ao evidente esgotamento dos combustíveis de origem fóssil, aos problemas ambientais gerados pela exploração, transporte e uso dos recursos naturais, à mudança climática, à contaminação, entre outros. $\mathrm{O}$ trabalho que aqui apresentamos, tem a intenção principal de propor um modelo conceitual baseado em uma construção teórica a partir de uma metodologia mista de análise documental, desde a formulação de três categorias: a educação em energias renováveis (EER), o compromisso público (CP) e a conceptualização elaborada pela nossa equipe sobre atitude energética ( $A E)$; tudo isto somado às necessárias interações que se geram desde a democracia participativa e a construção social comunitária que pretende envolver à sociedade como estrutura principal e não como um fim.

Em segundo lugar, a importância de contribuir ao desenvolvimento da área de pesquisa da Educação em Energias Renováveis, em razão das discussões que atualmente se planteiam frente à falta de responsabilidade social energética e o desinteresse pelo uso de energias alternativas como estratégia de redução dos problemas ambientais do contexto; por último, a necessidade de formular novos caminhos para a implementação do uso das energias renováveis por parte da cidadania com a intencionalidade de mitigar os problemas derivados de um desenvolvimento desmedido da ciência e da tecnologia, problemática que vem se incrementando nas últimas décadas. 
Palavras chave: atitude energética; compromisso público; educação em energias renováveis; modelo.

\section{Para citar este artículo:}

V. A. Ballesteros-Ballesteros, and A. P. Gallego-Torres, "Modelo de educación en energías renovables desde el compromiso público y la actitud energética," Revista Facultad de Ingeniería, vol. $28 \quad$ (52), $\quad$ pp. 2019. https://doi.org/10.19053/01211129.v28.n52.2019.9652.

Esta obra está bajo licencia internacional Creative Commons Reconocimiento 4.0

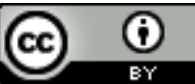




\section{INTRODUCCIÓN}

La crisis energética del planeta derivada de las graves consecuencias del abuso de los recursos naturales, se hizo evidente en la década los años ochenta del siglo pasado, donde se tomaron medidas como el ahorro energético y la transición a electrodomésticos y bombillas de eficiencia energética, pero los resultados no fueron los esperados, y este fue solo el inicio de un camino en el que se hizo necesario tomar otras medidas, que involucran procesos de innovación tecnológica en la producción, transformación, distribución y consumo de la energía. Lo que supuso un despliegue de tecnologías de energía renovable a gran escala, que suplieran el consumo global de energía de manera sostenible. Estos desarrollos dieron lugar a diferentes tipos de energías renovables tales como: energía eólica, energía solar, aplicaciones fotovoltaicas y térmicas, así como producción de biomasa, biogás, bioetanol y biodiesel. Sin embargo, estas medidas continuaron siendo insuficientes, debido fundamentalmente a los obstáculos epistemológicos y socioculturales, relacionados con mala difusión de las tecnologías de energía renovable, particularmente aquellas que involucran directamente a los ciudadanos, ya que en muchas ocasiones no satisfacen las necesidades percibidas de los usuarios finales, o para algunos no son rentables [1].

En la década de los noventa y gracias al trabajo de Lars Broman y Aadu Ott se inicia un nuevo paradigma en torno a la necesidad de mitigar la crisis energética del planeta, se trata de "La Educación en Energías Renovables" propuesto a partir de la publicación del artículo titulado: la educación en energía solar como camino a seguir, a partir del reconocimiento del efecto de la incorporación de distintos recursos didácticos en la enseñanza de la energía solar [2]; posteriormente, en el en el marco del Segundo Congreso Mundial de Energías Renovables, Lars Broman presentó un trabajo que destacaba la importancia de articular la educación en energía solar con las actividades relacionadas con la energía solar a nivel mundial.

Inicialmente, el paradigma educativo estaba enfocado al déficit de acceso al conocimiento sobre energía solar, así como también, a la falta de inclusión de los contenidos relacionados con las energías renovables en los currículos de todos los 
niveles de formación. Simultáneamente, se empezó a pensar en la EER como proyecto educativo multidisciplinar que involucrara la formación científica ciudadana y el desarrollo de actitudes y valores respecto a la energía y las energías renovables [3].

En éste sentido, se destaca que el tema energético debe ser abarcado como tópico fundamental en la enseñanza de las ciencias y las ingenierías, y con mayor importancia en una sociedad como la nuestra en la que se cuenta con una gran variedad de fuentes de energía, pero que sin embargo no se ha generado una conciencia de desarrollo que privilegie lo sustentable, de aquí surge la importancia de desarrollar un modelo de Educación en Energías Renovables que permita a las nuevas generaciones y a los ciudadanos en general una formación energética con el propósito que sean ellos quienes entiendan la problemática, generen una postura al respecto y se apropien de acciones y valores que ayuden a contribuir con el cambio necesario en la forma como nuestra sociedad concibe el uso y transformación de la energía [4].

Conceptualizar un modelo teórico y metodológico para la educación en energías renovables (EER) supone acudir a las elaboraciones que han venido configurándose alrededor del concepto de educación en energías renovables.

Elevar la EER a la categoría epistemológica de modelo supone comprender la realidad desde el compromiso público ascendente, de tal manera que permita el entendimiento del conjunto de elementos que componen el sistema y la relación jerárquica que existe entre ellos. Lo que nos ha llevado a la formulación de un nuevo concepto, el de actitud energética (AE), que desde ahora se configura como un elemento dinámico al establecer los conceptos frente a la predisposición de los ciudadanos hacia las energías renovables. Desde esta perspectiva se hace necesario determinar los elementos teóricos y metodológicos que nos permitirán modelar los constructos y dar un orden lógico al implementar, reconfigurar y sistematizar la validación de este nuevo concepto. 
El trabajo discute, en primer lugar, los elementos teóricos que son la base conceptual del modelo denominado CPAER; en segundo lugar, se describe de manera sucinta la metodología empleada en la construcción y validación del modelo; en tercer lugar, presentamos el modelo propuesto relacionado con la articulación entre el compromiso público, las actitudes, los comportamientos y las necesidades energéticas.

\section{ELEMENTOS TEÓRICOS DEL MODELO}

La formulación del modelo se plantea a partir de tres ejes conceptuales: la educación en energías renovables (EER), el compromiso público ascendente y la conceptualización de las actitudes energéticas (AE). En este sentido, y desde la epistemología socio constructivista planteada por Fourez, se asegura la confiabilidad en los escenarios y su permanencia en el tiempo.

\section{A. Educación en Energías Renovables (EER)}

La educación en energías renovables (EER) consiste en un imperativo permanente que promueve la concientización sobre la importancia de incorporar fuentes renovables no convencionales de energía y promover entre la ciudadanía el desarrollo de actitudes y valores que contribuyan a enfrentar los retos energéticos de las comunidades a través de prácticas educativas, social y culturalmente compatibles [4]. La EER se fundamenta a partir de los trabajos de Broman en 1984, cuando se inició la discusión sobre la aproximación didáctica que se dinamizó desde el conocimiento de objetos, es decir, tanto del qué enseñar, como de la metodología y la necesidad de incorporar medios y dispositivos para facilitar la enseñanza y el aprendizaje de las energías renovables [5]. En 1996, y con el ánimo se continuar aportando elementos de juicio a la discusión, se incorporó una línea de acción fundamental: la brecha existente entre oferta y demanda de energía; se destacó la necesidad de contar con personas capacitadas en los distintos niveles de formación, para alcanzar el éxito en la implementación de cualquier programa de formación en energías renovables [6]. A partir del año 2000, se traza un nuevo propósito: el Revista Facultad de Ingeniería (Rev. Fac. Ing.) Vol. 28 (52), pp. 27-42. Julio-Septiembre 2019. Tunja-Boyacá, 
desarrollo sostenible. A raíz de una preocupación creciente por las consecuencias del calentamiento global, se empezó a constituir una mirada multidisciplinaria de los problemas ambientales; se comenzó a vincular la tecnología desarrollada con los asuntos de política y economía energética y, muy importante, con los problemas sociales, de modo que avanza una concientización respecto a los desafíos ambientales que enfrenta la humanidad [7] [8].

\section{B. Compromiso Público (CP)}

El compromiso público consiste en la problematización de las dimensiones social y cultural del conocimiento científico y la consideración adecuada de las conceptualizaciones y valores públicos al respecto de las energías renovables durante las etapas iniciales de un desarrollo tecnológico [9] [10]. Pero ¿cuál es el rasgo fundamental de este concepto que nos permite plantear un modelo epistemológico? Sin lugar a duda, el talante característico del compromiso público ascendente es su énfasis en la participación del público (o comunidad) en forma "prospectiva" más que "retrospectiva" en decisiones que pueden afectarlo directa o indirectamente sobre la incorporación de nuevas tecnologías en su vida cotidiana [11]. En su configuración actual, la ciencia se encuentra bajo la presión de dos grandes crisis simultáneas y confluyentes: una se refiere a la confianza pública en la evidencia producida por la ciencia y sus instituciones; la otra se refiere a la gobernanza de la ciencia y la reproducibilidad e implementación de sus resultados [12] [13].

\section{Actitud Energética (AE)}

Desde la década de los 80 , o incluso antes, se estableció la importancia de incorporar en los modelos educativos el componente axiológico, con el fin de interpretar y entender los intereses y las actitudes de los estudiantes hacia la ciencia y su aprendizaje [14]. En este sentido y basados en la definición de Pozo [15] sobre actitud -que hace referencia a las tendencias o disposiciones adquiridas y Revista Facultad de Ingeniería (Rev. Fac. Ing.) Vol. 28 (52), pp. 27-42. Julio-Septiembre 2019. Tunja-Boyacá, 
relativamente duraderas sobre un objeto, fenómeno o situación al modelo propuesto por Fishbein y Azjen en 1995, que relaciona las actitudes, las creencias y la intención conductual, y al trabajo de Álvarez y Vega en 2009, quienes sostienen que la mayor parte de las investigaciones sobre las actitudes se centran en el valor predictivo que estas pueden tener sobre las conductas y que existen en la literatura muchos indicadores para medirlas y estudiarlas [16]. Hemos desarrollado un concepto estructurante que permita interactuar de manera dinámica en relación con las actitudes de los ciudadanos. Este término lo hemos denominado: "actitud energética", como la predisposición de los ciudadanos hacia el consumo, el ahorro, los comportamientos y las decisiones relacionadas con los desafíos energéticos. En este sentido, las actitudes energéticas están orientadas hacia la sensibilidad y la comprensión de los problemas energéticos actuales y el compromiso social de los sujetos con los recursos renovables y las energías no convencionales, pero, sobre todo, hacia la necesidad de establecer una responsabilidad social energética.

\section{MÉTOdO}

En esta sección se explicarán los aspectos centrales del diseño investigativo desarrollado, los cuales permitirán dar cumplimiento al objetivo planteado para el presente trabajo.

\section{A. Tipo de investigación}

La investigación se realizó a través de un diseño de tipo mixto cualitativo e interpretativo, de tipo documental, el cual determinó el procedimiento de selección, acceso y registro de la muestra documental. 


\section{B. Unidades de análisis}

A partir de categorías teóricas formuladas a priori, se construyó una matriz en Excel que determinó el procedimiento de selección, acceso y registro de la muestra documental y el análisis, interpretación y correlación entre las categorías. Por medio de Se reseñaron un total de 711 documentos, de los cuales por medio de la utilización del Atlas ti y las categorías, se escogió una muestra de 350 documentos.

\section{Procedimiento}

Una vez seleccionada la muestra se procedió a realizar un análisis del contenido de tal forma que se pudieran agrupar en las tres categorías: Educación en Energías Renovables, Compromiso Público, Actitudes Energéticas, realizar comparaciones y evitar repeticiones y redundancias en el proceso [17]. Este tipo de estudio se fundamenta desde el paradigma de la complejidad, lo que supone que los constructos y las formulaciones deben ser analizados desde diferentes perspectivas y deben obedecer a una reconfiguración y a la posibilidad de la implantación de categorías emergentes [18].

\section{Desarrollo}

En respuesta a los objetivos metodológicos, una vez establecida la base teórica y la necesidad de determinar líneas de acción que permitan el diseño, el desarrollo y la difusión de las energías renovables como parte de procesos sociales fundamentales para el mejoramiento de la calidad de vida de los seres humanos [19], nos dispusimos a la formulación y validación por parte de expertos de un modelo que responda a la educación comunitaria en energías renovables, bajo la conceptualización del compromiso público y las actitudes energéticas.

El propósito del modelo es generar un impacto ambiental positivo, en la medida que implica promover actitudes energéticas que permitan a los ciudadanos satisfacer las necesidades de energía, limitar los riesgos y la contaminación del aire, el agua, 
el suelo y la biosfera por la producción de energía; y, a su vez, contempla la formulación de escenarios y estrategias para la mitigación de los problemas energéticos, la responsabilidad social energética, la toma de decisiones y la oportunidad de elaborar proyectos, políticas y protocolos basados en las necesidades sociales y culturales del entorno geográfico donde se implemente.

El modelo se conjuga, como aparece a continuación, a partir de las categorías teóricas formuladas representadas de forma abstracta y dinámica a través del conjunto de interacciones que conceptual y metodológicamente se delimitan en torno al objeto de estudio: las energías renovables.

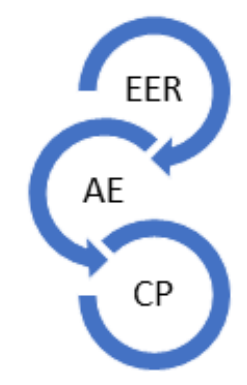

Fig. 1. Modelo CEPAER.

Como se observa, la estructura del modelo es dinámica, los hechos o fenómenos que enlaza de manera inferencial posibilitan el ordenamiento de dichos fenómenos. Siendo el compromiso público como base ascendente del proceso de construcción de conocimiento desde los sujetos el que determina ese ordenamiento y no un orden que se supone se halla en la naturaleza de lo ya establecido como base teórica y como escenario tecnológico.

A partir de la representación icónica del modelo, el trabajo subsecuente consistió en la formulación de los componentes dinámicos y su estructura conceptual, que sugiere un marco de referencia que permita sistematizar los resultados y sus posteriores reelaboraciones.

El modelo cumple con la categoría epistemológica, dado que une de manera inferencial las proposiciones que afirma sobre los conceptos que en él se integran. De esta forma, su estructura es diferente debido a su carácter dinámico y a la 
interrelación de sus estructuras. El modelo así concebido, impulsa la inteligibilidad y ayuda a la comprensión de los fenómenos, ya que proporciona los canales de interconexión entre hechos que sin la existencia de los lazos inferenciales podrían permanecer aislados e in

dependientes unos de otros [20].

La descripción detallada del modelo, el cual ha sido validado por expertos, se encuentra a continuación:

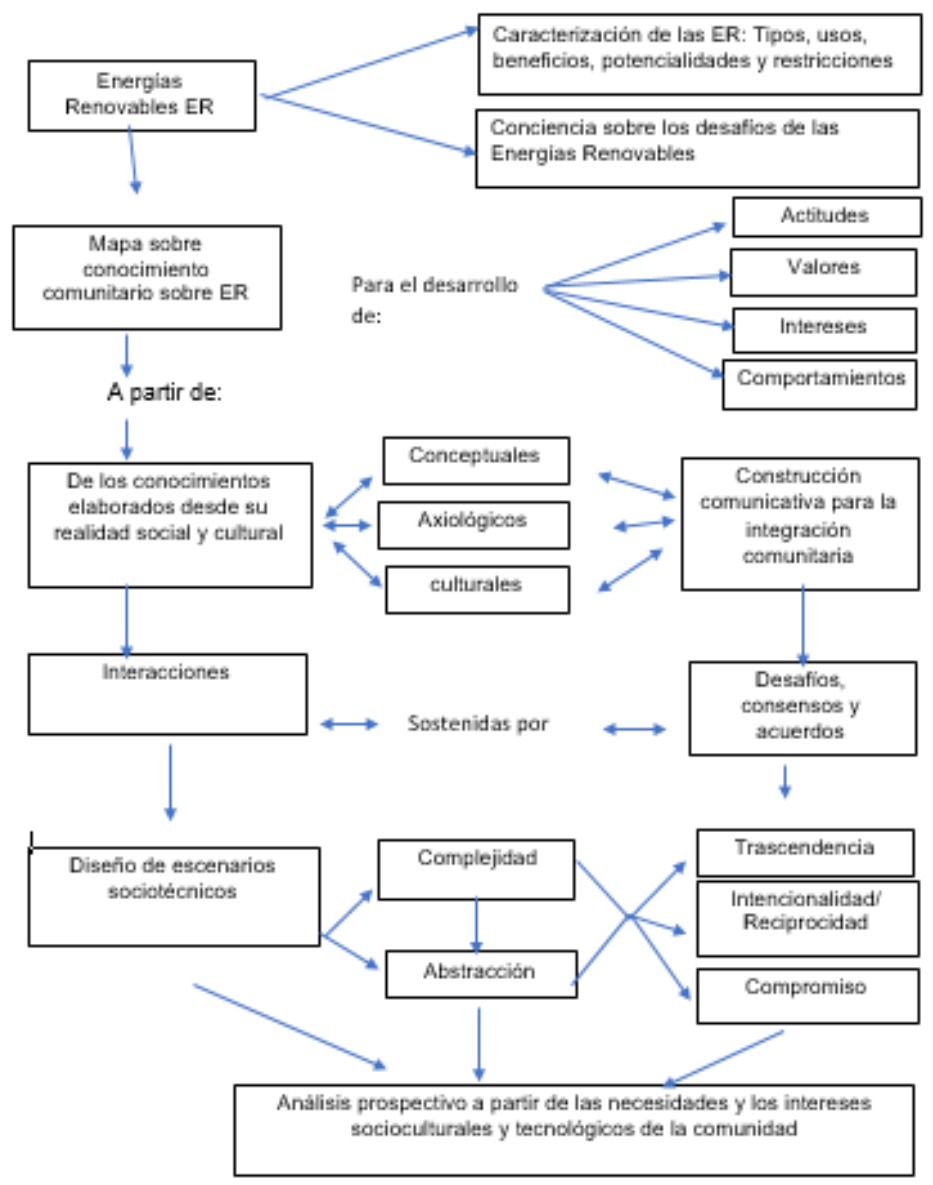

Fig. 2. Modelo CPAER.

El modelo teórico propuesto, configurado a través de tres ejes fundamentales, EER$A E$ y $C P$, pretende integrar exitosamente un programa de apropiación de energías Revista Facultad de Ingeniería (Rev. Fac. Ing.) Vol. 28 (52), pp. 27-42. Julio-Septiembre 2019. Tunja-Boyacá, Colombia. L-ISSN: 0121-1129, e-ISSN: 2357-5328, DOI: https://doi.org/10.19053/01211129.v28.n52.2019.9652 
renovables como proyecto educativo comunitario, con el propósito de promover el compromiso público ascendente con esta tecnología. Además de acercar las energías, el modelo apunta a nuevas posibilidades en el desarrollo de trayectorias de ciencia y tecnología más receptivas a las necesidades de desarrollo comunitario. Muestra un anhelo real de fortalecer la participación en la ejecución de objetivos científicos que respondan a los problemas sociales y ambientales; pero, al mismo tiempo, presenta una evaluación deprimentemente cruda de su propia impotencia para contribuir a dicha agenda.

La apertura de nuevas formas de participación es un desafío clave para el gobierno nacional, regional y local; para los profesores, que deben estar preparados para estos nuevos retos de mediación; y para la comunidad en general, que deberá integrarse a los debates nacientes y al despliegue tecnológico con el propósito de mejorar sus condiciones básicas de saneamiento.

\section{Conclusiones}

En la actualidad, el fenómeno global de cambio climático está a la vanguardia de las preocupaciones internacionales, por lo que la energía es considerada un tema muy especial y se requiere con urgencia estudiar todas sus dimensiones detalladamente. De ahí la necesidad de establecer procesos de educación formal e informal comunitaria, mediante la implementación del modelo propuesto, donde se construya conocimiento con fundamento en sus intereses, necesidades y actitudes energéticas, que permitan mitigar los déficits energéticos y el agotamiento de los recursos energéticos de origen fósil y la contaminación producto de las otras formas de energía.

\section{CONTRIBUCIÓN DE LOS AUTORES}

Los autores contribuyeron de igual manera en el desarrollo de la investigación y en la escritura del artículo presentado. 
Modelo de educación en energías renovables desde el compromiso público y la actitud energética

\section{REFERENCIAS}

[1] T. C. Kandpal, and L. Broman, Renewable Energy Education for the Future. Sweden: Strömstad Akademi, 2016.

[2] L. Broman, and A. Ott, "Solar education: the way forward," Sun at Work in Europe, vol. 6, pp. 24-25, 1988.

[3] H. Garg, and T. Kandpal, "Renewable energy education in developing countries: Indian scenario," Renewable Energy, Technology and the Environment, vol. 3, p. 2430-2438, 1992.

[4] V. Ballesteros-Ballesteros, "La educación en energías renovables como alternativa de promoción del compromiso público ascendente entre los Indígenas Wayuu en la Alta Guajira," Revista Científica, vol. 2 (Número Especial), pp. 388-397, 2019.

[5] T. Kandpal, and H. Garg, "Renewable Energy Education for Technicians/Mechanics," Renewable Energy, vol. 16 (1), pp. 1220-1224, 1998. https://doi.org/10.1016/S0960-1481(98)00488-1.

[6] H. Garg, and T. Kandpal, "Renewable Energy Education in Developing Countries: Indian Scenario," Renewable Energy, Technology and the Environment, vol. 3, pp. 2430-2438, 1992. https://doi.org/10.1016/B978-0-08-041268-9.50015-8.

[7] M. González, J. Gonçalves, and R. Vasconcelos, "Sustainable Development: Case Study in the Implementation of Renewable Energy in Brazil," Journal of Cleaner Production, n. 142, pp. 461-475, Jan. 2017. https://doi.org/10.1016/.j.jclepro.2016.10.052.

[8] S. Gyamfi, N. Derkyi, E. Asuamah, and I. Aduako, "Chapter 6 - Renewable Energy and Sustainable Development," Sustainable Hydropower in West Africa, pp. 75-94, 2018. https://doi.org/10.1016/B9780-12-813016-2.00006-X.

[9] M. Carapuço, R. Taborda, M. Silveira, and C. Andrade, "Upstream Public Engagement on Coastal Issues: Audience Response to a Science-Based Exhibition," Ocean \& Coastal Management, n.. 144 , pp. 83-89, Jul. 2017. https://doi.org/10.1016/i.ocecoaman.2017.04.008.

[10] M. García, and J. A. López-Cerezo, "Pájaros y ornitólogos. Una conversación sobre el pasado, el presente y el futuro de la relación ciencia-filosofía," CTS Revista Iberoamericana de Ciencia, Tecnología y Sociedad, vol. 10 (28), pp. 159-166, 2015.

[11] T. Rogers-Hayden, and N. Pidgeon, "Developments in Nanotechnology Public Engagement in the UK:'Upstream' towards Sustainability?" Journal of Cleaner Production, vol. 16 (8-9), pp. 1010-1013, May. 2008. https://doi.org/10.1016/i.jclepro.2007.04.013.

[12] A. Saltelli, and S. Funtowicz, "What Is Science's Crisis Really About?" Futures, vol. 91, pp. 5-11, Aug. 2017. https://doi.org/10.1016/j.futures.2017.05.010.

[13] J. R. Porter, and B. Wollenweber, "Science in an Age of (Non) Reason," In: Progress in Science, Progress in Society, pp. 59-70, 2018. https://doi.org/10.1007/978-3-319-69974-5 6.

[14] A. Robles, J. Solbes Matarredona, J. Cantó Doménech, and L. Lozano, "Actitudes de los estudiantes hacia la ciencia escolar en el primer ciclo de la Enseñanza Secundaria Obligatoria," Revista Electrónica de Enseñanza de las Ciencias, vol. 14 (3), pp. 361-376, 2015.

[15] I. Pozo, Aprendices y maestros, la nueva cultura del aprendizaje. Madrid: Alianza, 2000.

Revista Facultad de Ingeniería (Rev. Fac. Ing.) Vol. 28 (52), pp. 27-42. Julio-Septiembre 2019. Tunja-Boyacá, Colombia. L-ISSN: 0121-1129, e-ISSN: 2357-5328, DOI:

https://doi.org/10.19053/01211129.v28.n52.2019.9652 
[16] P. Álvarez Suárez, and P. Vega Marcote, "Actitudes ambientales y conductas sostenibles. Implicaciones para la educación ambiental," Revista de Psicodidáctica, n.․14, pp. 25-38, 2009.

[17] M. Gómez-Vargas, C. Galeano-Higuita, and D. Jaramillo-Muñoz, "El estado del arte: una metodología de investigación," Revista Colombiana de Ciencias Sociales, vol. 6 (2), pp. 423-442, Jul. 2015. https://doi.org/10.21501/22161201.1469.

[18] A. Adúriz-Bravo, and A. Gallego-Torres, "Scientific Research and its Pedagogical Uses," Revista Científica, vol. 2 (25), pp. 160-161, Aug. 2016. https://doi.org/10.14483//udistrital.jour.RC.2016.25.a0.

[19] T. C. Kandpal, and L. Broman, "Renewable Energy Education: A Global Status Review," Renewable and Sustainable Energy Reviews, vol. 34, pp. 300-324, Jun. 2014. https://doi.org/10.1016/j.rser.2014.02.039.

[20] R. Gallego-Badillo, "Un concepto epistemológico de modelo para la didáctica de las ciencias experimentales," Revista Electrónica de Enseñanza de las Ciencias, vol. 3 (3), pp. 301-319, 2004.

[21] R. Gallego-Torres, and P. González-Crespo, "Metodología de la investigación en ingeniería," Revista Científica, vol. 2 (29), pp. 5-7, 2017. https://doi.org/10.14483/23448350.11959.

[22] M. Fishbein, and I. Ajzen, Belief, Attitude, Intention and Behavior. Boston: Addison Wesley, 1975. 